\title{
Trombocitopenia y aplasia radial (TAR) de curso benigno en gemelas univitelinas
}

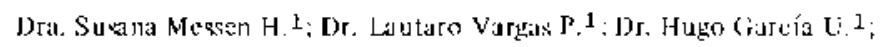 \\ Dra. Patriciá Miranda $6 i^{2}$
Congenital Thrombocytopenia and absent radius syndrome in identical twins

\begin{abstract}
Congenital thrombocytopinta and absent radius syndromi (TAR) was found in two identibl twins whilu-have becis followed trom the newbon period up to t]e des of six years. Both patients have thrumbocytopenia, typueal skeletal abnormalities, initial myeloid leukemoid reaction. bone marrow eosimoplilia and paucity of megakaryocytes. Their thinical picture is remarkably ujld with abxence of purperat and only slight blecdings. No reforence to TAR in identical twans were found in a review of eurrenl literature, but its ocurrente in univitelun twins argues in lavour of a genetical or jegr for the diseres.

(Key words: Tlırombocytopenia, congenital, abset)t radius, identical twins!.
\end{abstract}

El síndrome de trombocitopenia asociada a aplasia radial (TAR) es hereditario, se presenta esporádicamente $y$ se asocia con un trastorno 'hematolúgico (trombucitopenia amegačariociti(e) y defectos esqueléticos (ausencia bilateral de los radios) ${ }^{1}, 2,3,4,5,6$. Hasta 1981 se hah ialn publicado alrededor de 50 casos en el mundo y sólo un caso en cl país.

En la Linidad de Iematología Pediátrica del Hospital San Juan de Dios, liemos tenido la oportunidad de estudiat dos gemelas con TAR, detectado ers el período de recién nacido por el hallazgo de alterationes esqueléticas caracteristicas y confirmado modiante estudio hematológico. El curso clínico de nuestros casos tra sido benigno. lo que no es habitual de acuerdo con la información disponible, $y$ csta es. al parecer, la primera vez que se comurica sobre trombouitoponia asociada a aplasia rádial (TAR) en gemelos univitelinos.

\section{Casos Clínicos}

Dos genelas univitelinas fueron estudiadas on la unidad de hematología del servicio de Pediatría del Hospital San Juan de Dios y seguidas

1. Servicio de Pediatría, Iospital San Juan de Dios. Departamento de Pediatría División Cicneias Médicas: Oceidente. Facultad de Medicina, tiniversidad de Chile.

2. Becaria del Servicio de Pediatria, Hospital Luis Calvo Mackenna. División Ciencias Médieas Oriente, Mateultad de Medicina, Universidad de ('li ile. desde el nacimiento hasta la edad de 6 años. (Fig. 1 A y 1 B). Ambas nacicron de parto cutócico el 20 de Marzo de 1979 (KRC y PRC). Sus pesos de nacimiento fueron 2.650 y $2.750 \mathrm{~g}$ $y$ sus tallas 47 y $48 \mathrm{~cm}$. respectivamente. El cmbarazo habia sido normal y no habian antecedentes de ingestión de drogas. No habian antecedentes familiares de consunguinidad, ni enfermedades genéticas. Los padres y tres herma. nos eran sanos. Ambas nifías tenían al nacer maxcada hipoplasia de ambos antebrazos, desviación de las manos en sentido radial, clinodactilia bilatcral y pulgares presentes. El resto del examen físico era normal (Ver Figs. I y 2). Los recuentos de plaquetas dieron respectivamente, cifras de $38.000 \times \mathrm{mm}^{3}$ y $34.000 \times \mathrm{mm}^{3}$. En los mielogramas realizados a la edad de un mes la celularidad era normal, había discreta eosinofilia, la proporción de megacariocitos estaba disninuida, los megacariocitos tenian pocos gránulos y carecian de plaquetas en su periferia. Un año después los mielogramas mostraban respectivimente las proporciones celulares de la Tabla 1

Despues de revisar varios frotis se pudicron detectar escasos megacarioblastos: 20 y $40 \%$; megacarjocitos basófilos: 30 y $10 \%$; megaciriocitos granulosos: 50 y $50 \%$. en la mayoria con 3 ó más núcleos. No se encontraron megacariocitos plaquetarios.

Las radiografias (Figura 2) ausencia bilateral de los radios, acortamiento del antebrazo, desviación de las manos en dirección radial, hipoplasia cubital bilateral y clinodactilia bilateral. La edad ósea estaba retrasada, con disociación 

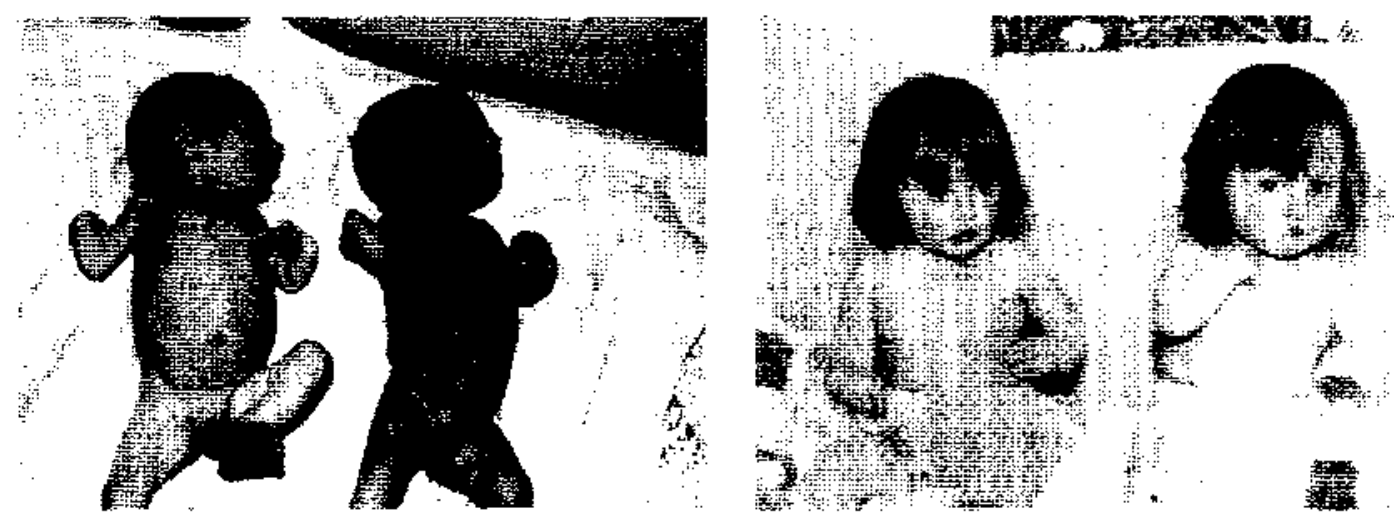

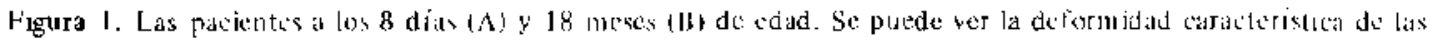
exitemilades saperiors call wala por aplasia radial.

Tabla $\mathbf{I .}$

Publiciones ciutares (\%) t'tl al miclogratma de dos pacientes (KRC y PRC) con T tombocilopenial y aples siat radial al la codad de un año.

\begin{tabular}{|c|c|c|}
\hline CILLILA & KIरC' & PRQ \\
\hline Micloblasios & 3 & 2.5 \\
\hline Promiclocjos ne:utrólilos & 4 & 3 \\
\hline Mielocilus neutrólilos & 15.5 & 17,5 \\
\hline Mieferitos cosimólides & 1.5 & 2,5 \\
\hline Juveniles neutrúfilos & 4,5 & 17,5 \\
\hline Juven iles cosinotifilos & 2.5 & 1.0 \\
\hline Batcjiformes ncutrólitos & 11.5 & 14,5 \\
\hline Potinucleares neutrólilus & N.D. & 10,0 \\
\hline Baciliformes eosinotilos & N.I. & 0,5 \\
\hline Polinuclestes conjuófilos & 5,5 & 3,5 \\
\hline Linfociton & $\therefore . D$. & 15,0 \\
\hline $\begin{array}{l}\text { Mera cariocitos } \\
\text { (e } 200 \text { célulass }\end{array}$ & 0 & 0 \\
\hline
\end{tabular}

N.D. = No desctitos

metacarpo falángica (los huesus del carpo con edad ésea inferior a los del retacarpa). El resto del estudio esquelét ico tile normal.

Ambas fueron tratadas con yalyas de yeso y férulas de plástico en los antcorazos pira corregir la desviación radial, prednisona 0,5 a $1 \mathrm{mg} x$ $\mathrm{kg} x$ día por períodos de 3 meses como máximo. cuando el recuento plaquetario disminuyo de 30.000 plaquetas $\times \mathrm{mm}^{3}$ y sulfato ferroso. por bajo peso de nacimicnto y gemelaridad. Después de la edad de unl año se les har dado prednisona y har sido seguidas con contrules cada ve\% más espaciados.

Las pacientes no han sufrido hemorragias espontáneas tii púrpura. KCR tuvo una hemorritgia oral post -contusional. Ambas tonían talla normal al año de edad y léficit de peso año de $15 \%$ (KRC) y $13 \%$ (PRC). $\Lambda$ los 6 años ambas gemelas tenúan déficit pondo-estatural leve (15\%), con desarrollo psicotnotor normal.

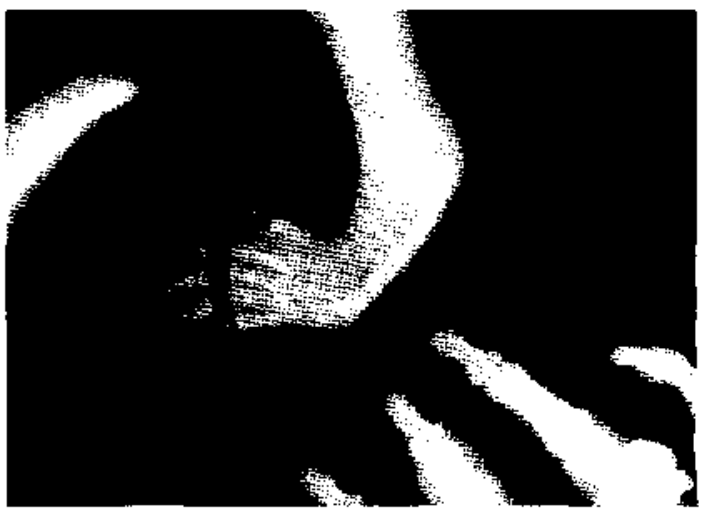

ligura 2. K.R.C. (A) y P.R.C. (B) ausentia del radio, lipoplissia colvital y clinodatetilia en las radiogratlials de strentidat superior.

\section{EVOLUCION PLARUETAS EN GENELAS UNIVITEUNAS CON TAR}

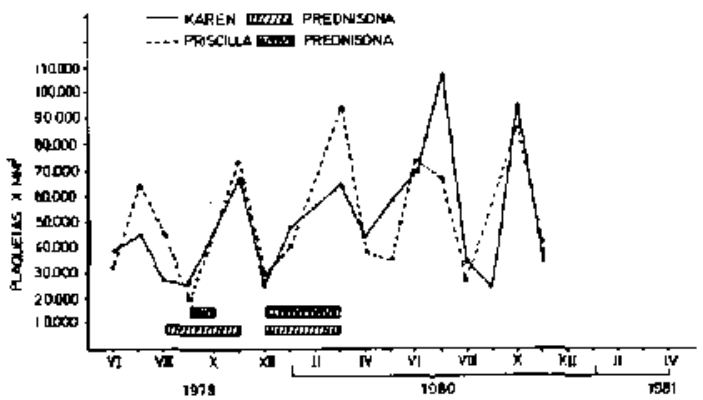

Figura 3. Se observa trombocitopenia oxcolante en ambus gemeles durante los prineros 18 meses. Curya de evolución del recuento de placjuetas en las dos gemelas tou sinfrome de tromboctloponia y aphasia radial.

Lat evelución de la trombocitopenia ha sido oscilante (Fig. 3) con eosinofilias absolutas máximas de 1.640 (KRC) y 2.875 (PRC), leucocitosis con reacción leucemoide que persistió liastu el cuarto mes de vida. 


\section{COMENTARIO}

El defecto esquelétido del TAR fue descrito por primera vez por Kato en $1924^{7}$. BirchJensen informó una incidencia de aplasia radial de $1 \times 30.000$ nacidos vivos ${ }^{8}$. Esta malformación esquelética se asocia en $40 \%$ de los casos con otras anomalías; cardiopatías congénitas $(10,6 \%)$; espina bifida, paladar hendido, labio leporino $(4,7 \%)$; alteraciones renales $(4,7 \%)$ y alteraciones gastrointestinales $3,5,6,8,9,11,12,13,14$. Nuestros casos sólo tenján malformaciones esqueléticas. La asociación de malformación esquelética (ausencia de radios) y púrpura trom. bocitopénico amegacariocítico comenzó a ser descrita en la década del $50^{2,15,16}$ y recibió el nombre de sindrome TAR (trombocitopenja aplasia radio) en $1969^{4}$. En $78 \%$ de estos pacientes existen también alteraciones de los cúbitos (ausencia bilateral, unilateral o hipoplasia), que también puede ocurrir en manos, búmeros, escápula $(75 \%)$; piernas y pies $(40 \%)$; tronco (18\%) y caderas $(5 \%)^{4,8,14}$. La edad ósea está atrasada en todos los casos.

La otra gran manifestación de este síndrome es el púrpura trombocitopénico atnegacariocítico. En muchos casos aparecen sangramientos de piel y mucosas desde las primeras horas de vida; en $95 \%$ éstos ocurren antes de los 4 meses de edad ${ }^{1,13,17,18}$ pero se ha descrito disminu. ción de la tendencia al sangramiento desde el $6^{\circ}$ mes de vidal,14. El espectro clínico de las hemorragias es muy amplio, desde pocas petequias a intenso púrpura y aún mâs, hemorragia cerebral o digestiva de curso fatal. Alrededor de $40 \%$ de los enfermos de TAR fallecen durante el primer año de vida ${ }^{14,18}$. La trombocitopenia sueje fluctuar entre 5.000 a 30.000 o más plaquetas por $\mathrm{mm}^{3}$ con bruscas caidas a menos de 10.000 por $\mathrm{n}^{3} \mathrm{~m}^{3}$. Se desconoce la causa de estas acentuaciones de la trombocitopenia, mancionándose en la literatura que los sufrimientos de distinto origen, infecciones, intervenciones qujrúrgicas, alergia a la leche de vaca pueden pre. ceder a su aparición $1,14,20$. Algunos autores describen aumentos del número de plaquetas después del primer año de vida ${ }^{1}$. La función plaquetaria en el TAR es normal, pero se ha descrito su asociación, en un caso, con trombocitopatia y en otro con enfermedad del depósito de plaquetas 21,22 .

La concentración de leucocitos en la sangre periférica suele variar entre 10.000 y 30.000 por $\mathrm{mm}^{3}$, con neutrofilia ${ }^{23}$, reacción leucemoide $y$ recuentos que exceden los 40.000 por $\mathrm{mm}^{3}$ en el $50 \%$ de estos pacientes. Se ha descrito eosinofilia relativa y absoluta en el $53 \%$ de los casos. La basofilia también se observa con frecuencia ${ }^{14}$. La serie roja es habitualmente, normal. Algunos casos aislados han evolucionado con poiquilocitosis, policromatofilia y anisocitosis 23,24 y en dos casos se ha descrito microesferocitosis $y$ aumento leve del índice hemolitico ${ }^{25}$, La alteración más importante en el mielograma, es la disminución de los megacarjocitos que faltan totalmente en $66 \%$, están disminuidos en $12 \%$ o son inactivos en el $12 \%$ restante de estos enfer. mos. Los megacariocitos contienen pocas granu. laciones, son poco lobulados y algunos presentan vacuolas en el citoplasma 26 .

En las gemelas que hemos descrito las hemoragias fueron minimas, posiblemente porque el recuento plaquetarjo se ha mantenido, generalmente, por encima de las cifras consideradas peligrosas, oscilando entre 19.000 a $107.000 \mathrm{x}$ $\mathrm{mm}^{3}$. En ambas la reacción leucemoide desapareció alrededor del $4^{\circ}$ mes.

El retardo del desarrollo pondo-estatural con mayor compromiso de la talla y el peso de nacimiento inferior al percentil 25 de las tablas de Meredith, han sido previamente descritos en el TAR, como también función intelectual normal, como en nuestras pacientes. Sin embargo, se han descrito alteraciones neurológicas y retraso mental como secuelas de hemorragias cerebrales ${ }^{14}$.

Considerando la existencia de malformaciones de los miembros superiores, TAR debe ser diferenciado de la anemia de Fanconi, la embriopatía por talidomida y el sindrome de Holt Oram, pues todas estas condiciones pueden tener hipoplasia o aplasia radial. La anemia de Fanconi se caracteriza por hipoplasia o aplasia de los pulgares: en el TAR siempre están los cinco dedos; el compromiso hematológico del sindrome de Franconi es más tardío y afecta a las tres series hematopoyéticas ${ }^{27}$. En la embriopatía por talidomina las lesiones consisten en focomelia, sin alteraciones hematológicas. El síndrome de Holt Oram se caracteriza por la asociación de malformación del pulgar y cardiopatía congenita, sus alteraciones hematológicas y de herencia autosómica dominante ${ }^{6}$.

El tratamiento del TAR es sintomatico. Se utilizan transfusiones de concentrados de plaquetas durante las crisis de trombocitopenia que se acompañan de hemorragias o antes de intervenciones quirúrgias ${ }^{28}$. La esplenectomia se ha intentado ocasionalmente con resultados discutibles ${ }^{14,24,29}$. El emplẹo de esterojdes disminuye la tendencia a sangrar pero no tiene efectos beneficiosos sobre la trombocitopenia $16,18,28$. El tratamiento ortópédico tiene como propósito corregir las desviaciones de la mano y mejorar su función con yesos, férulas, correajes e incluso intervenciones quirúrgicas 13,14 . El pronóstico de los pacientes con TAR es malo, $40 \%$ mueren en 
el primer año de vida como consecuencia de bemorragias digestivas o cerebrales ${ }^{14}$. Con e] transcurso de los años la mortalidad disminuye ya que las alteraciones hematológicas y las hemorrágicas son menos intensas ${ }^{1422}$, alcanzando los sobrevivientes, las mismas expectativas de yida que la población general. De esto deriva la im. portancia de hacer oportunamente el diagnóstico y el manejo correcto en las primeras etapas de la vida: se puede reconocer el TAR in el feto por nedio del ultrasonido y de radiografías obstétricas cuando existen antecedentes familiares de $\mathrm{TAR}^{5}, 26,27,32,33,34,45$.

En nuestros pacientes llama la atención la benignidad del síndrome pues prácticamente no presentaron hemorragias ni púrpura y su trombocitoperia fue más bien moderada. Sólo encontramos una referencia al TAR en nuestro pais ${ }^{38}$ y ninguna en gemelos univitelinos. En la actualidad se supone que el TAR es un síndrome genético que se hereda en forma recesiva $1,5,14$, 15,27 , pudiendo ocurrir más de un caso en la misma familia.. Algunos casos en hermanos que avalaл esta presunción $6,24,27$ argumento que sería reforzado por nuestra experiencia, sin embargo no es posible descartar el efecto de una noxa externa como han postulado otros autores ${ }^{1}, 13,27,29$. Además se ha presentado evidencia relacionando TAR con la embriopatía de la rubéola ${ }^{30.39}$ y la administración de tiazidas durante el embarazo 40

\section{RESUMEN}

Se describen dos gemelas con síndrome de trombocitopenia asociada a aplasia radial (TAR) que fueron seguidas desde el nacimiento hasta los seis años de edad. Ambas tenian trombocitopenia, alteraciones esqueléticas típicas, reacción leucemoide inicial, eosinofilia medular y disminución de los megacariocitos medulares, pero a diferencia de otros pacientes presentaron una evoluciôn más bien benigna. Los autores no encontraron referencias a este síndrome en gemelos univitelinos, hecho que aparece respaldar la tesis de que el TAR es un problema de origen genético.

\section{REFERENCIAS}

1. Nilsson, L.R., Lumdholm, G.: Congenital thrombocytopenja associated with aplasia of radious. Acta Paediatr. Scand. 49: 291, 1960.

2. Emeryl, J.L., Gordon, R.R., Rendte-Short, J., Varadi, S., Warrack, A.J.N.: Congenital amegakariocytic thrombocytopenia with congenital deformities and leukemoid blood pictura in the new born. Blood. 12: $567,1957$.
3. Robson, H.N., Walker, CH.M.: Congenital and noonit tal thrombocytopenic purpura. Atch. Dis Child. 26: 179,1951 .

4. Hall, J.G., Levin, J., Kuh, J.P., Ottenheimer, E.J., Von Berhum, K.A.P., Mc Kusick, I.A.: Thrombocytopenia with absent radius (TAR). Medicine 48 : 4) l, 1969.

5. Ruthy, Mack, Hirsch, J.L.: Cheng, E.: Prenatal ultrasound diagnosis of thrombocytopenia with absent radii. Am. J. Obstet, Gynecol, 141: 350, 1981.

6. Smith, D.M. Atlas de Malformaciones Somáticas en el niño. pp 132-137 Ediciones Pediátricas, Barcelo $\mathrm{na}, 1972$.

7. Kato, $K$.: Congenital absecne of the tadiaus with review of literatura and report of three cases, $J$. Bone. Joint. Surg. 6: 589, 1924.

8. Birch-Sensen, A.: Congenital deformities of the upper extremities. Fd. F.jлar Munh ggard Furles, Copenhugen, 1949.

9. Poznarski, $A . K$. . The hand in radiologic diagnosis. Shortening of absense of portions of the hands and digites Ed. W.B. Saunders Company, London 1974 , The Series Saounders Monography in Clinical Radiologic Vol. 4: pp. 176- 180.

10. Simcha, $S$. Congenital Heart Disease in R.adial clubbed hand syndrome. Arcit. Dis. Child. 46:345, 1971 .

11. Von Landolt, R.F.: Kongenitale (Neonatal) Thrombopenicn. Helv. Paediarr. Actt 3: 3, 1048.

12. Greenwald, H.M., Shernan, I.: Congenital essential thrombopenia. Am. J. Lis. Child. 38: 1245, 1929.

13. Holt, M., Orom. S.: Familial heart disease with skelctal malformations. Br. Heirt, J. 22: 236, 1960.

14 Fayen, H.: Thrombocytopenia with absent radii (The TAR síndiome). Am. J. Med, Sei. 280: 95 , 1980.

15. Shaw. S, Otiver, R.A.M.: Congenital Hypoplastic thrombocytopenia with skeletal deformities in siblings. Blood. 14: 374, 1959.

16. Gross, H., Groh, CH., weippl, G.: Kongenital hypoţlastische thrombopenic mit radiusaplasic neuc. Oesternz Kinderheil K. 1: $574,1956$.

17. Elliot, M., Eisenstein, C.: Congenital Amegakaryocytic thombocytopenic purpura. Clin. Pediatr. 3: 143, 1966.

18. Dignan, P., Mauer, A.M.: Mechanisms of Myeloid loukemoid reaction associated with skeletal abnormalities. J. Pediat. 63: 939, 1965.

19. Pearson, H., Mi intosh, S: Veonatal Thrombocytopenia. Clin. Haematol. 7: 111, 1978.

20. Whilfield, M.F., Bar, D.G.D.: Cow's milk allergy in the Syndrome of Thrombocytoperia with absent radius. Arch. Dis. Child. 5: 337, 1976.

21. Sultan, Y.M.L. Schobohach, Let et at: Abnormal platelet function, population and survival time in a boy with congenital absent radii and thrombocytopenia. Lancet. 2: 653, 1972.

22. James Day, M.D., Holm Holsem, Ph.D.: Platelet aderine nucleotide "Storage Pool Deficiency" in thrombocytopenic absent radii Syndrome. JAMA $221: 28,1972$.

23. Dignen, P. Mater, A.M., Frantz. C.: Phocomelia with congenital hypoplastic thrombocytopenia and myeloid leukemoid reactions. J. Pediatr. 70: 561 . 1967.

24. Smith, C.: Thrombocy topenia with absent radius. Congenital hypoplastic thrombocytopenia, primary amegakaryocytic thrombocytopenia phocomelia and congenital hypoplastic thrombocytopenia, 
myeloid leukemoid reaction (TAR). Blood Diseases of Infancy and Childhood pag. 782. The C.V. Mosby Co., 1972.

25. Zetterstrom, $\boldsymbol{R}$, Strinberg, B.: Sporadic Congenital Spherocytosis associated with congenital hypoplastic thrombocytopenja and malfomations. Acta Paediatr. Scand. 47: 14, 1958.

26. Victor, K., Lui, M.D., Abdelsalam, H. Ragab, M.D. Bone marrow cultures in children with Fanconi Anemia and the T.4R Syndromc. J, Pcdiatr. 80: 952,1977 .

27. Nitssor. L.R.: Chronic pancytopenia with multiple congenital abnormalities (Fanconi's anaemia). Acta Paediatr. 49: 518, 1960.

28. Bell, S.D., Mold, J,W., Otrier, R.A.M., Shaw, S.: Study of transfused platelets in a case of conge nital hipoplastic thrombocytopenia. Br. Med. J. 2: 692, 1956.

29. Stefanini, M., Domeshek, W': The hemorrhagic disorders: A Clinical and Therapeutic Approach. Grune \& Stratton, New York, Pp, 106-107, 1955.

30. Hugh-Jones, K., Manfield, P.A., Brewer, H.F.: Congenital thrombocytopenic purpura. Arch. Dis. Child. 35: 146, 1950.

31. Skerick, S.K., Flot, A.E.: The anatomy of congenital radial dysplasia. Its surgery and functional implications. Clin. Orthop. 66: 12S, 1969.
32. Mahoney, M.M.D., Hobbins, J.: Prenatal Diagnosis of Chondroectodermal Dy splasia (Ellis-Van Cleveld Syndrome) with fetoscopy and ultrasound. New Engl. J. Med. August 4, 1977.

33. Mac-Mahon, B, Levy, M.A.: Prenatal Origin of Childhood Leukemia. N. Engl. J. Med. 270: 1082, 1964.

34. Stewart, A.M., Webb, J.W., Hewitt, D.: A survery of childhood matignancies. Br. Med. J. 1:,1495. 1958.

35. Stewarl, A.M., Kneale, G.W.: Radiation dose effects in relation to obstetric $x$-rays and childhood cancers. Lancet, 1: 1185, 1970.

36. Omenn, G.S., Figley, M.M., Graham, C.B. et al: Prospects for radiographic intrauter inc diagnosis the syndrome of thrombocytopenia with absent radii. N. Engl. J. Med, 288: 7?7, 1973.

37. Omenn, G.S., Hall, J.G., Graham, C.B, et al: The use of radiographic visualization for prenatal diagnosis. Birth Defects 12: 217, 1977.

38. Borlone, R.: Trombocitopen ia con radios ausentes TAR. Rev. Chil. Pediati. 48: 258, 1977.

39. Berge, T., Brumnhage. F., Nisson. L.R.: Congenital hypoplastic thrombocytopenia in rubelk cmbriopathy. Acta Pacdiatr. 52: 349, 1963.

40. Rodriguez, S.D., Leiken, S.L., Hiller, M.C.: Neonatal thrombocytopenia associated with anterpartum administration of thiazide drugs. N. lingl. J. Med. 270: 881,1964 . 